\title{
A Bayesian approach to the probability of coronary heart disease subject to the -308 tumor necrosis factor- $\alpha$ SNP
}

\author{
Ekaterini Vourvouhaki ${ }^{1 *}$ and C. Sofia Carvalho ${ }^{2,3 \dagger}$ \\ 1 Department of Science in Dietetics and Nutrition, Harokopio University of Athens, \\ Eleutheriou Venizelou 70, GR 17671 Athens, Greece \\ 2 Instituto de Plasmas e Fusão Nuclear, Instituto Superior Técnico, \\ Av. Rovisco Pais, 1, 1049-001 Lisboa, Portugal and \\ 3 Academy of Athens, Research Center for Astronomy and Applied Mathematics, \\ Soranou Efessiou 4, 11-527, Athens, Greece
}

(Dated: October 20, 2018)

\begin{abstract}
We study the correlation of the occurrence of coronary heart disease (CHD) with the presence of the single-nucleotide polymorphism (SNP) at the -308 position of the tumor necrosis factor alpha (TNF- $\alpha$ ) gene. We also consider the influence of the occurrence of type 2 diabetes (t2DM). Using Bayesian inference, we first pursue a bottom-up approach to compute the working hypothesis and the probabilities derivable from the data. We then pursue a top-down approach by modelling the signal pathway that causally connects the SNP with the emergence of CHD. We compute the functional form of the probability of CHD conditional on the presence of the SNP in terms of both the statistical and biochemical properties of the system. From the probability of occurrence of a disease conditional on a given risk factor, we explore the possibility of extracting information on the pathways involved in the occurrence of the disease. This is a first study that we want to systematise into a comprehensive formalism to be applied to the inference of the mechanism connecting the risk factors to the disease.
\end{abstract}

\footnotetext{
* Email: kvourvouhaki@gmail.com

† Email: carvalho.c@gmail.com
} 


\section{INTRODUCTION}

We are interested in the association of diseases, in particular of coronary heart disease (CHD), with genetic factors in order to determine underlying genetically-driven functional mechanisms that are causally related to the disease. In this context, environmental factors are regarded as contaminants. Among the risk factors for the emergence of CHD, genetic determinants may provide a wealth of information on the nature of the disease, which can be used to develop new diagnosis and treatment methods. The study of these factors has attracted the effort of many research teams for the identification of disease susceptibility genes as well as acquired somatic mutations. Among these genes is that of the tumor necrosis factor alpha (TNF- $\alpha$ ), a pleiotropic cytokine produced mainly by macrophages and T-cells which is involved in the inflammatory response of the immune system [1].

It has been suggested that the TNF- $\alpha$ gene affects the modulation of lipid metabolism, obesity susceptibility, and insulin resistance [2 4], thus being potentially implicated in the development of cardiovascular diseases (see Ref. [5] and references therein). Several singlenucleotide polymorphisms (SNPs) have been identified in the human TNF- $\alpha$ [6, 7]. The best documented of these SNPs are at position -308 of the TNF- $\alpha$ gene promoter. It involves the substitution of guanine $(\mathrm{G})$ for adenine $(\mathrm{A})$ and the creation of two alleles, TNF1(A) and TNF2(G), and three genotypes, GG, GA and AA [8]. There is evidence implicating TNF- $\alpha$ in an increased susceptibility to the pathogenesis of a variety of diseases (see Ref. [4] and references therein). However, the results on its association with CHD are contradictory, some implying different influence of the two alleles on the prevalence of CHD [3, 4], others implying no association [9-12]. This conflict is due in part to the results being based on the frequentist analysis [13].

In order to infer the risk of CHD derived from potential risk factors, it is important to develop a formalism that extracts all possible information from the data and combines them with other data sets on different intervening factors for a consistent inference of the correlations. Here we introduce a possible formalism based on Bayesian inference and test its applicability on the three-variable data set from Ref. [2]. In this manuscript we attempt to quantify the risk of occurrence of CHD based on its association with the SNP at the position -308 of the TNF- $\alpha$ promoter. This entails the calculation of a probability distribution for the occurrence of CHD conditional on the SNP or other factors. The influence of other factors is here illustrated by the occurrence of type 2 diabetes (t2DM).

When, instead of computing the probability distribution of some quantity produced by the process, we compute the conditional probability of an unsolved variable in the process given the observed variables, we are solving an inverse probability problem. This requires the use of the Bayes theorem. The Bayesian approach has been used extensively for parameter inference and model selection from cosmology [14-16] to biology [17-20] among many others. In this case, we observe the occurrence of a given disease and the correlation with a SNP. In

the absence of a theory, we relate the SNP with the disease via a model of the potentially 
implicated pathways. The parameters of this model are the relevant factors that we want to infer from the data. Such a theory would be important as a first step to predict the occurrence of a genetically-driven disease for a given polymorphism, as well as to understand the mechanism of genetic mutation from which polymorphisms derive. In this study we propose a solution to the first problem and will approach the second problem in a forthcoming study.

The causal relation between the risk factors and the occurrence of the disease is a function of the rates which characterize the implicated pathways. Here, knowing how the occurrence of the disease is distributed over the parameter space of the risk factors and knowing how the risk factors act at the biochemical level, we show how we can extract information on the pathway involved in the emergence of the disease. However, since the pathways involved are most likely interconnected with others, sourced by different factors, the next step would be to allow for the participation of various factors in the emergence of the same disease. There is a plethora of sparse phenomenological/symptomatic data on the simultaneous occurrence of SNP's and diseases from which correlations are tentatively drawn. The formalism developed here can be extended to other factors in the effort to systematise the sparse data to identify risk factors, to combine them into a comprehensive model for the mechanism that leads to the disease and therefrom to infer a universal law of gene mutation.

The manuscript is organized as follows. In section II we select the working hypothesis for the relation between the SNP and CHD on the basis of the Bayes factors and compute the probabilities in the presence of the SNP derivable from the data. One of these will be used as the likelihood for the occurrence of CHD. In section III we suggest a simplistic model for the signalling pathway between the onset of the SNP and the emergence of CHD, and compute the posterior probability for the occurrence of CHD. Finally we comment on the results and indicate the research routes that we will be exploring next.

\section{BOTTOM-UP APPROACH}

We will base our analysis on the data reported in Ref. [2], which consist of frequencies of occurrence of CHD as a function of the SNP at the position -308 of the TNF- $\alpha$ promoter. It is also advanced a correlation between the SNP and an increased predisposition to CHD in type 2 diabetic patients. That study also comprises an analysis of the gender dependence on diabetes.

The sample of CHD patients consists of $N_{C H D}=341$ randomly selected patients. Out of these, $N_{C H D, t 2 D M}=106$ also suffered from t2DM. Another sample of type 2 diabetic patients numbering $N_{\overline{C H D}, t 2 D M}=135$ was selected among non-CHD patients. These two samples,

together with a control sample of $N_{\overline{C H D}, \overline{t 2 D M}}=207$ non-CHD non-diabetic patients, were analysed for the occurrence of the -308 TNF- $\alpha$ SNP. Thus the total number of diabetic patients consists of a random and a non-random component on the factor CHD. Since we 


\begin{tabular}{ccccc} 
& \multicolumn{2}{c}{$C H D$} & \multicolumn{2}{c}{$\overline{C H D}$} \\
\cline { 2 - 5 } & $t 2 D M$ & $\overline{t 2 D M}$ & $t 2 D M$ & $\overline{t 2 D M}$ \\
\hline$S N P$ & 43 & 67 & 26 & 48 \\
$\overline{S N P}$ & 63 & 168 & 109 & 159 \\
\hline
\end{tabular}

Table I: The data. Frequencies of the TNF $\alpha-308$ SNP in CHD patients, t2DM patients and controls.

are interested in studying the correlation between the SNP and CHD, we cannot use the data on the sample of non-CHD diabetic patients to extract information on the frequency of occurrence of CHD given that diabetes had occurred, because the information would be biased. We can, however, derive information on the frequency of diabetes given that the SNP or CHD occurred. The data are summarized in Table I.

\section{A. Model comparison: the Bayesian evidence}

Given the data, we can derive the influence of CHD and t2DM on the SNP. We have three variables, namely occurrence of CHD $(C H D)$, occurrence of t2DM $(t 2 D M)$ and presence of the SNP $(S N P)$, and six hypotheses for the presence of the SNP. The hypotheses are the following: $H_{00}$ : the probability of the SNP does not depend on the occurrence of either CHD or t2DM; $H_{01}$ : the probability of the SNP depends on the occurrence of CHD; $H_{10}$ : the probability of the SNP depends on the occurrence of t2DM; $H_{11}$ : the probability of the SNP depends on the independent occurrence of both CHD and t2DM; $H_{11}^{\text {chd }}$ : the probability of the SNP depends on the occurrence of CHD and on the occurrence of t2DM given that CHD is present; $H_{11}^{t 2 d n}$ : the probability of the SNP depends on the occurrence of t2DM and on the occurrence of CHD given that diabetes is present. These are schematically depicted in Fig. 1

We note that, given the selection criterion for the population of $N_{\overline{C H D}, t 2 D M}$, we cannot use the corresponding data to infer on the occurrence of CHD given the occurrence of t2DM since they would bias the results. For this reason, $H_{11}^{t 2 d m}$ is excluded as a viable hypothesis given the data collected. We proceed to compare the remaining hypotheses based on Bayesian evidence. The probability of an hypothesis given the data is the posterior probability of the corresponding model [21]

$$
P\left(H_{i} \mid D\right)=\frac{P\left(D \mid H_{i}\right) P\left(H_{i}\right)}{P(D)}
$$

where $P\left(D \mid H_{i}\right)$ is the evidence, $P\left(H_{i}\right)$ is the prior probability of $H_{i}$ and $P(D)=$ $\sum_{i} P\left(D \mid H_{i}\right) P\left(H_{i}\right)$. In order to infer which hypothesis is more likely in view of the data, we compare the evidence computed for the alternative hypotheses. The evidence is the 


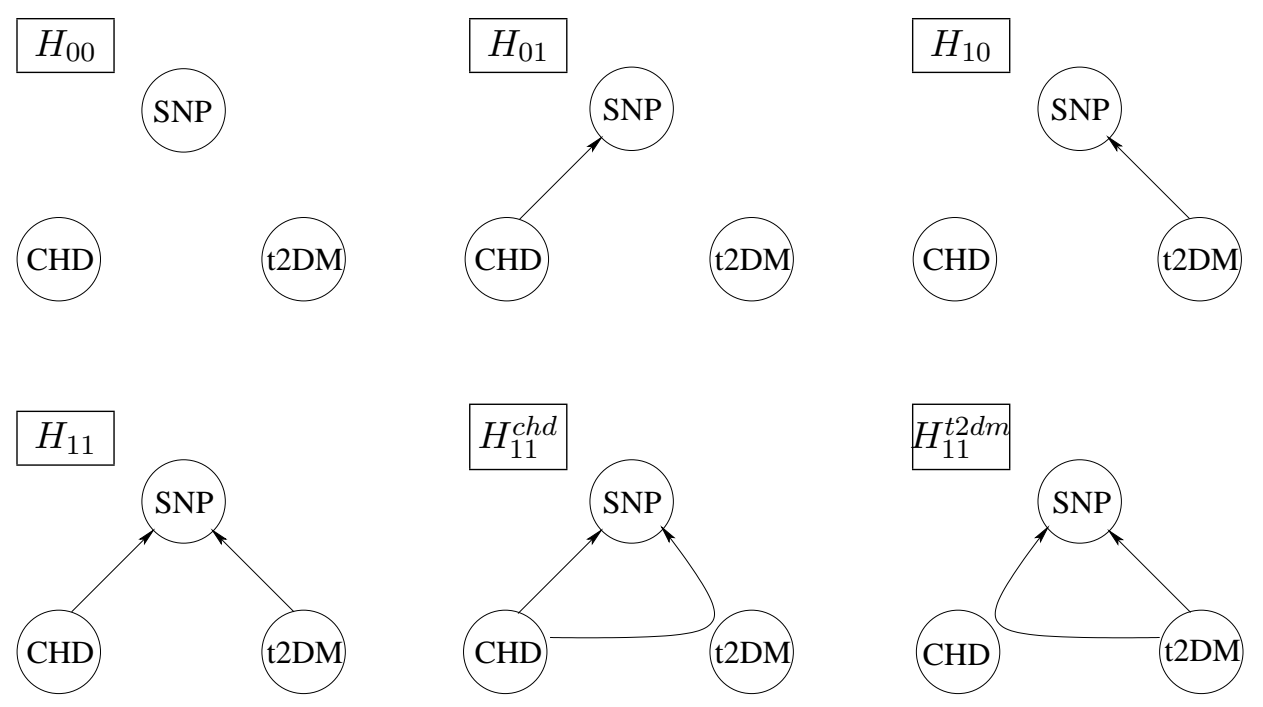

Figure 1: Diagram of the hypotheses considered. The circles denote the corresponding variables. The arrows denote a correlation between the encircled variables which they connect. A straight arrow indicates that the probability of the variable at the tip of the arrow is conditional on the variable at the origin of the arrow. A curved arrow indicates that the probability of the variable at the tip of the arrow is conditional on the variable at the inflection of the arrow, which moreover is conditional on the variable at the origin of the arrow. In the upper set we have $H_{00}$, $H_{01}$ and $H_{10}$; in the lower set we have $H_{11}, H_{11}^{c h d}$ and $H_{11}^{t 2 d m}$.

integral of the likelihood over the parameter space $\theta$ of the model

$$
P\left(D \mid H_{i}\right)=\int d \theta P\left(D \mid \theta \wedge H_{i}\right) P\left(\theta \mid H_{i}\right)
$$

Assuming equal prior probabilities for the different hypotheses, then

$$
\frac{P\left(H_{i} \mid D\right)}{P\left(H_{j} \mid D\right)}=\frac{P\left(D \mid H_{i}\right)}{P\left(D \mid H_{j}\right)} .
$$

We compute the evidence for the five hypotheses described above (for details see Appendix A). In order to compare the hypotheses, we take the logarithm of the ratio of the corresponding evidences, $P\left(H_{i} \mid D\right) / P\left(H_{j} \mid D\right)=B_{i j}$, which we present in Table II. This quantity is known as the Bayes factor and gives empirical levels of significance for the strength of the evidence. It also encapsulates Occam's factor which measures the adequacy of the hypothesis to the data over the parameter space of the hypothesis [21. The levels of significance ascribed to the Bayes factor are calibrated by the Jeffrey's scale [22] as follows: if $1.0<B_{i j}<3.2, H_{i}$ should not be favoured over $H_{j}$; if $3.2<B_{i j}<10$, there is substantial evidence for $H_{i}$ over $H_{j}$; if $10<B_{i j}<100$ there is strong evidence, while for $B_{i j}>100$ the evidence for $H_{i j}$ should be considered decisive. In the first column, we find the Bayes factors which relate each hypothesis with $H_{00}$. Since hypothesis $H_{00}$ describes the data as the result of a random process, this column measures the preference for a departure from randomness [23]. From these values we infer that all hypotheses are substantially favoured 


\begin{tabular}{c|ccccc}
$B_{\text {row,col }}$ & $H_{00}$ & $H_{01}$ & $H_{10}$ & $H_{11}$ & $H_{11}^{\text {chd }}$ \\
\hline$H_{00}$ & 0 & & & & \\
$H_{01}$ & 3.99 & 0 & & & \\
$H_{10}$ & 4.37 & 0.91 & 0 & & \\
$H_{11}$ & 4.08 & 1.02 & 0.93 & 0 & \\
$H_{11}^{\text {chd }}$ & 7.07 & 1.77 & 1.62 & 1.73 & 0 \\
\hline
\end{tabular}

Table II: The Bayes factors for the hypotheses considered. Here $B_{\text {row }, \text { col }}=H_{\text {row }} / H_{\text {col }}$.

over $H_{00}$. The values seem to suggest that $H_{11}^{c h d}$ is also favoured over the other hypotheses, however they are not sufficient to infer substantial evidence. Since $H_{11}^{\text {chd }}$ is the hypothesis that exhibits the most substantial evidence over the null hypothesis (this hypothesis was supported in Ref. [2] with $p=0.0056$ ), we will take $H_{11}^{\text {chd }}$ as our working hypothesis upon which we will base our subsequent inferences.

\section{B. Model fitting}

Having inferred from the computation of the evidence which of the possible hypotheses is most likely to be compatible with the data in the presence of the SNP, we proceed to compute the probability for the occurrence of the polymorphism. Let $H$ denote our working hypothesis. Then the probability that the SNP is present is

$$
P(S N P \mid H)=\int d \theta P(S N P \mid \theta) P(\theta \mid D \wedge H)=\int d \theta P(S N P \mid \theta) \frac{P(D \mid \theta \wedge H) P(\theta \mid H)}{P(D \mid H)} .
$$

Since $H_{11}^{\text {chd }}$ consists of a two-component hypothesis [24], each of which described by two parameters, the resulting parameter space is four-dimensional. The equation above must be generalized for a multidimensional parameter space where each factor is no longer a scalar but instead a $(4 \times 4)$ matrix. Since each component consists of two disjoint sets, the matrix is diagonal, each component being weighted by the relative size of the population. We then write

$$
P(S N P \mid H)=\int d^{2} p P(S N P \mid p) P(p \mid D \wedge H)+\int d^{2} \bar{p} P(S N P \mid \bar{p}) P(\bar{p} \mid D \wedge H) .
$$

Here the indices range over the two-dimensional parameter spaces, with $p=\left(p_{01}, p_{11}\right)$ and $\bar{p}=\left(p_{0 \overline{1}}, p_{\overline{1} 1}\right)$, where for simplicity we have dropped the tilde from the notation used in Appendix A. In particular, $p_{01}$ is the frequency of SNP given the occurrence of CHD and $p_{0 \overline{1}}$ the frequency of SNP given non-occurrence of CHD, both subject to non-occurrence of t2DM, whereas $p_{11}$ is the frequency of SNP given that t2DM has occurred and $p_{\overline{1} 1}$ the frequency of SNP given that t2DM has not occurred, both subject to CHD having occurred. Let $P(S N P \mid p)=p$ and $P(S N P \mid \bar{p})=\bar{p}$. The posterior probability of $p$ is by the Bayes 
theorem

$$
P(p \mid D \wedge H)=\frac{P(D \mid p \wedge H) P(p \mid H)}{P(D \mid H)}=\frac{P(D \mid p \wedge H)}{P(D \mid H)}
$$

and similarly for the posterior probability of $\bar{p}$. In the last step we assume for simplicity a uniform prior for both $p$ and $\bar{p}{ }^{1}$ Writing the evidence as

$$
P(D \mid H)=\int d^{2} p P(D \mid p \wedge H) P(p \mid H)+\int d^{2} \bar{p} P(D \mid \bar{p} \wedge H) P(\bar{p} \mid H)
$$

we find for hypothesis $H_{11}^{c h d}$ that

$$
\begin{aligned}
& P(S N P \mid H)= \\
= & \frac{1}{P(D \mid H)} \int d p_{01} \int d p_{11} \int d p_{0 \overline{1}} \int d p_{1 \overline{1}} \\
\times & {\left[\begin{array}{c}
N_{C H D, \overline{t 2 D N}} \\
N_{S N P, C H D, \overline{t 2 D N}}
\end{array}\right) p_{01}^{N_{S N P, C H D, \overline{t 2 D N}}+1}\left(1-p_{01}\right)^{N_{\overline{S N P}, C H D, \overline{t 2 D N}}} } \\
& +(1-\gamma)\left(\begin{array}{c}
N_{C H D, t 2 D N} \\
N_{S N P, C H D, t 2 D N}
\end{array}\right) p_{11}^{N_{S N P, C H D, t 2 D N}+1}\left(1-p_{11}\right)^{N_{\overline{S N P}, C H D, t 2 D N}} \\
& +\tilde{\gamma}\left(\begin{array}{c}
N_{\overline{C H D}, \overline{t 2 D N}} \\
N_{S N P, \overline{C H D}, \overline{t 2 D N}}
\end{array}\right) p_{0 \overline{1}}^{N_{S N P, \overline{C H D}, \overline{t 2 D N}}+1}\left(1-p_{0 \overline{1}}\right)^{N_{\overline{S N P}, \overline{C H D}, \overline{t 2 D N}}} \\
& \left.+(1-\tilde{\gamma})\left(\begin{array}{c}
N_{C H D, \overline{t 2 D N}} \\
N_{S N P, C H D, \overline{t 2 D N}}
\end{array}\right) p_{\overline{1} 1}^{N_{S N P, C H D, \overline{t 2 D N}}+1}\left(1-p_{\overline{11} 1}\right)^{N_{\overline{S N P}, C H D, \overline{t 2 D N}}}\right]
\end{aligned}
$$

which yields

$$
\begin{aligned}
P(S N P \mid H)= & \frac{1}{P(D \mid H)} \\
\times & {\left[\gamma \frac{N_{S N P, C H D, \overline{t 2 D N}}+1}{\left(N_{C H D, \overline{t 2 D N}}+2\right)\left(N_{C H D, \overline{t 2 D N}}+1\right)}\right.} \\
& \left.+(1-\gamma) \frac{N_{S N P, C H D, t 2 D N}+1}{\left(N_{C H D, t 2 D M}+2\right)\left(N_{C H D, t 2 D M}+1\right)}\right] \\
& +\tilde{\gamma} \frac{N_{S N P, \overline{C H D}, \overline{t 2 D N}}+1}{\left(N_{\overline{C H D}, \overline{t 2 D N}}+2\right)\left(N_{\overline{C H D}, \overline{t 2 D N}}+1\right)} \\
& \left.+(1-\tilde{\gamma}) \frac{N_{S N P, C H D, \overline{t 2 D N}}+1}{\left(N_{C H D, \overline{t 2 D M}}+2\right)\left(N_{C H D, \overline{t 2 D M}}+1\right)}\right] .
\end{aligned}
$$

Here $\gamma=N_{C H D, \overline{t 2 D M}} / N_{C H D}$ and $\tilde{\gamma}=N_{\overline{C H D}, \overline{t 2 D M}} / N_{\overline{t 2 D M}}$, with $P(D \mid H)$ given by Eqn. (A9). Substituting the values from Table I] we find that $P(S N P \mid H)=0.30 \pm 0.001$, which we identify as the effective mutation rate $\lambda_{\text {eff }}$ of the Poisson probability distribution describing the occurrence of the SNP. Although comparable, this value is different from the naïve guess

\footnotetext{
${ }^{1}$ This choice of prior is justified by the absence of an a priori bias on the values of these parameters.
} 
$\lambda=N_{S N P} / N=0.27$ or from the more elaborate one arising from the assumption of the null hypothesis [see Appendix $B$ for the derivation]. It then follows that the posterior probability for the occurrence of $n$ mutations in a population of size $N$ is

$$
P\left(n \mid \lambda_{e f f} \wedge N\right)=\exp \left[-\lambda_{e f f} N\right] \frac{\left(\lambda_{e f f} N\right)^{n}}{n !} .
$$

Similarly we compute

$$
\begin{aligned}
P(S N P \mid C H D \wedge H)= & \int d^{2} p P(S N P \mid C H D \wedge p) P(p \mid D \wedge H)=\int d^{2} p \frac{p P(D \mid p \wedge H)}{P(D \mid H)} \\
= & \frac{1}{P(D \mid H)}\left[\gamma \frac{N_{S N P, C H D, \overline{t 2 D N}}+1}{\left(N_{C H D, \overline{t 2 D N}}+2\right)\left(N_{C H D, \overline{t 2 D N}}+1\right)}\right. \\
& \left.+(1-\gamma) \frac{N_{S N P, C H D, t 2 D N}+1}{\left(N_{C H D, t 2 D M}+2\right)\left(N_{C H D, t 2 D M}+1\right)}\right]
\end{aligned}
$$

and find that $P(S N P \mid C H D \wedge H)=0.20 \pm 0.001$. We can also compute

$$
\begin{aligned}
P(t 2 D N \mid C H D \wedge H) & =\int d p P(t 2 D M \mid C H D \wedge p) P(p \mid D \wedge H) \\
& =\frac{1}{P(D \mid H)} \frac{N_{C H D, t 2 D N}+1}{\left(N_{C H D}+2\right)\left(N_{C H D}+1\right)}
\end{aligned}
$$

finding that $P(t 2 D N \mid C H D \wedge H)=0.09 \pm 0.001$. The errors indicated were computed from error propagation, assuming the error of a counting result $n$ to be $1 / \sqrt{n}$.

\section{TOP-DOWN APPROACH}

We now proceed to estimate the influence of the SNP on the occurrence of CHD. We want to find the posterior probability of the occurrence of CHD given the presence of the SNP, i.e.

$$
P(C H D \mid S N P \wedge H)=\frac{P(S N P \mid C H D \wedge H) P(C H D \mid H)}{P(S N P \mid H)} .
$$

Here $P(C H D \mid H)$ is the prior probability of CHD and $P(S N P \mid C H D \wedge H)$ is the likelihood of CHD for a fixed SNP. The remaining term $P(S N P \mid H)$ has no CHD dependence and can thus be absorbed into the normalization constant. It is known as the evidence or the marginal likelihood.

\section{A. A simplistic model for the signalling pathway}

Since the working hypothesis relates the presence of the SNP with both the occurrence of CHD and the occurrence of t2DM, we infer that the changes from the canonical pathway 
(a)
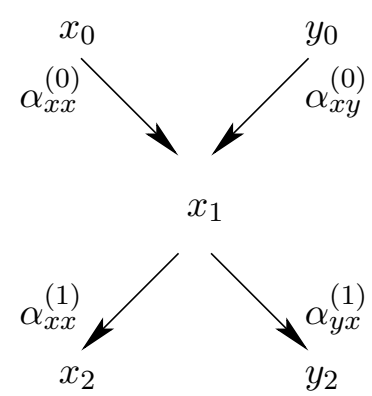

(b)

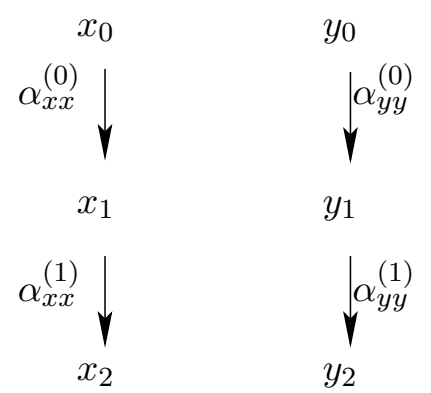

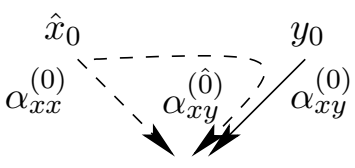
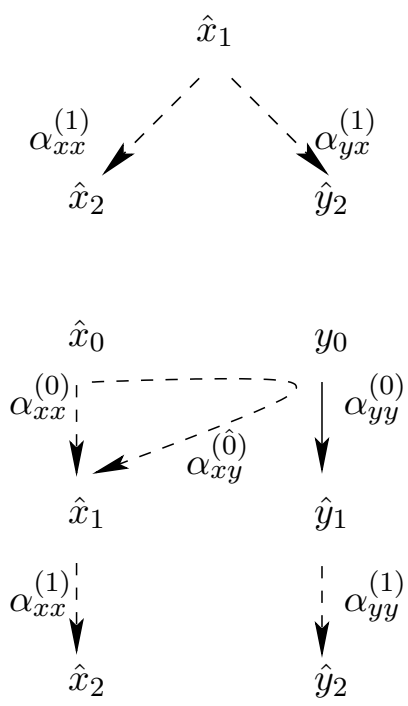

Figure 2: Diagram of the pathways with different source signals. The first column depicts the original architecture corresponding to the canonical pathway (i.e., in the absence of the SNP). The second column depicts one possible alteration of the corresponding pathway due to the SNP. Here the SNP acts on the signal $x_{0}$. The continuous lines represent the functioning pathways, and the dashed lines represent pathways which can suffer delay or cease to function entirely (in the case of previously existing pathways), or which could simply not come to exist (in the case of newly generated pathways). (a) Case 1a). For $y_{0}=0$ this reduces to Case 2. (b) Case 1b).

introduced by the SNP will have repercussions on the signalling cascades which regulate the emergence of CHD and t2DM. If we assume that the SNP will only affect one source signal, then this correlation suggests that the resulting signal transduction pathways interfere with one another. Functional interference can be derived from a common source signal or from common components downstream [25, 26]. As far as the source signal is concerned, we allow for two possibilities: 1) the pathways have different source signals; 2) the pathways have the same source signal and sufficiently downstream diverge. In case 1) and in order to reproduce interference between the two signalling pathways we can still have two further sub-cases: 1a) the pathways share components and the effect of the SNP consists of either an alteration in the velocity of the affected signal or an alteration of one of the pathways; 1b) the pathways do not share components but the pathway altered by the SNP shares components with the unaltered one. These two subcases can be distinguished by the correlation between the two diseases in the absence of the SNP, with case 1a) describing the existence of an a priori correlation between the occurrence of the two diseases and case 1b) the absence of such correlation. In case 2) the interference is built-in so the effect of the SNP is similar to that in case 1a). The diagrams are depicted in Fig. 2 which we proceed now to describe.

The variables $x$ and $y$ describe black boxes along the pathways that regulate the emergence 
of $\mathrm{CHD}$ and t2DM respectively. By black boxes we mean unresolved chemical reactions where no intervening elements are specified other than the input and the output reaction rates between two sequential black boxes. The index of the variables denotes the relative position in the pathway of the corresponding black box component. Thus '0' indicates the upstream component fed by the initial signal and which is subject to alteration upon the action of the SNP, whereas '2' indicates the final component which determines the emergence of the disease, with ' 1 ' denoting the intermediary component where the interference of the altered pathway with the unaltered one is manifested. Here the SNP affects the signal $x_{0}$ and propagates downstream through an altered or newly created pathway ' $\hat{0}$ '. A variable which refers to an altered pathway is denoted by $\hat{x}_{0}$, whereas a variable which refers to a newly created pathway is denoted by $x_{\hat{0}}$. The same rule holds for the corresponding rates. The cases where the original pathway ceases to function can be interpreted as inhibition. This a simplistic description of the potential signalling pathways involved, which is but a caricature of the real biochemical system. Nonetheless it is the possible description based on the data, which moreover captures the functional correlations inferred.

To select the working model we use as criterion the possibility of the emergence of a connection between the SNP and each disease without the participation of the other. We conceive three possible ways for the SNP to function: (1) the signal does not suffer any alteration from that in the absence of the SNP; $(2)$ the signal is triggered at a smaller rate so that $\hat{x}_{0}<x_{0} ;(3)$ no signal is triggered. We can exclude (1) on the basis that it contradicts the working hypothesis. Moreover, since (3) can be described as a limiting case of (2) when $\hat{x}_{0}=0$, we proceed to solely analyse (2) with each suggested model according to the disease combinations which can be reproduced.

Case 1a) If both $x_{2}$ and $y_{2}$ require that $x_{1}$ be acted by the products of both $x_{0}$ and $y_{0}$, then the functional time scales of the two pathways should be very close. In the case of a slower reaction rate, if the signalling pathways are assumed to consist of an isolated system then both diseases would occur. The way to prevent it would be by capturing the required reagents from neighbouring pathways. This scenario, however, would be beyond our current capabilities of inference and constraint. If instead $x_{1}$ has the flexibility of being independently activated by the two signals and of also independently acting upon $x_{2}$ and $y_{2}$, then the emergence of the diseases will depend on the supply of each pathway that develops from $x_{1}$ downstream. Should a single triggering signal be enough to supply for both downstream pathways, it could be the case that no disease occurs. This would moreover depend on the phase difference between the generation of $x_{1}$ upon the activation of $x_{0}$ and $y_{0}$. Should one triggering signal not be enough, then either disease could occur. This could be prevented if a compensation mechanism were triggered so that, in the absence of an effective signalling from one source, the working source would be stimulated according to the deficiency.

Case 1b) In this case, $x_{1}$ and $y_{1}$ are independently sourced and independently develop their pathways downstream. In the case of a slower reaction rate, no interference would be 
generated as long as the SNP-affected pathway could still run. However, in the case of a more drastic reduction of the trigger of $x_{1}$, an alternative pathway would be intercepted and the required reagents deviated. This could lead to a case of competition. Should pathway $x$ be thus maintained, pathway $y$ could either collapse or continue, resulting in the disease implicated in the pathway $y$ emerging or not. If pathway $x$ cannot be maintained and the corresponding disease is not avoidable, then we can still have either emergence or not of the other disease, depending on the degree of reagent deviation.

This empirical analysis can be complemented by the following quantitative one. We will assume that the system under study can be described as a dynamical one. In the absence of the SNP, the dynamical system is described by the rates indicated in Fig. 2, first column. If the SNP has occurred in the coding of the input signal in $x_{0}$, then the system will instead be sourced by the altered $\hat{x}_{0}$ and described by the rates as indicated in Fig. 2, second column.

We compute the fixed points (identified by the superscript ' $*$ ') of the quantities involved in both the canonical pathway and the SNP-altered one. The fixed points describe the state of the system in dynamical equilibrium and are computed by setting the time derivates equal to zero. Since we are interested in the probabilities which describe the average states of the system and not in the dynamical evolution that leads to those states, the fixed points are the variables to be used.

For case 1a)

$$
\begin{aligned}
\frac{d \hat{x}_{1}}{d t} & =\alpha_{x x}^{(0)} \hat{x}_{0}+\left[\alpha_{x y}^{(0)}+\alpha_{x y}^{(0)} \beta\left(\hat{x}_{0}\right)\right] y_{0}-\left(\alpha_{x x}^{(1)}+\alpha_{y x}^{(1)}+\delta_{x}^{(1)}\right) \hat{x}_{1} \\
\frac{d \hat{x}_{2}}{d t} & =\alpha_{x x}^{(1)} \hat{x}_{1}-\delta_{x}^{(2)} \hat{x}_{2} \\
\frac{d \hat{y}_{2}}{d t} & =\alpha_{y x}^{(1)} \hat{x}_{1}-\delta_{y}^{(2)} \hat{y}_{2}
\end{aligned}
$$

where the $\delta$ 's denote decay rates. Here $\beta$ is the fraction of the output of $y_{0}$ which activates $x_{1}$ and thereby attempts to compensate for the deficiency of activation of $x_{1}$ derived from the SNP

$$
\beta\left(\hat{x}_{0}\right)=\left(1-\frac{\hat{x}_{0}}{x_{0}-\Delta_{0}^{\text {thres }}}\right) \Theta\left[x_{0}-\Delta_{0}^{\text {thres }}-\hat{x}_{0}\right]
$$

where $\Theta$ is the Heaviside step function. This term is reminiscent of the carrying capacity term which sets an upper limit to cellular growth [27]. Here we set a lower limit, as determined by $\Delta_{0}^{\text {thres }}$, to the deficiency in $x_{0}$ caused by the altered signal for the onset, as imposed by the step function, of the compensating mechanism. This quantity would measure a functional marker for the presence of the SNP. We compare the fixed points in both the canonical pathway and the SNP-altered one, finding that

$$
\begin{aligned}
& \hat{x}_{2}^{*}-x_{2}^{*}=\left[\alpha_{x x}^{(0)}\left(\hat{x}_{0}-x_{0}\right)+\alpha_{x y}^{(\hat{0})} \beta\left(\hat{x}_{0}\right) y_{0}\right] \frac{\alpha_{x x}^{(1)}}{\delta_{x}^{(2)}} \frac{1}{\alpha_{x x}^{(1)}+\alpha_{y x}^{(1)}+\delta_{x}^{(1)}} \\
& \hat{y}_{2}^{*}-y_{2}^{*}=\left[\alpha_{x x}^{(0)}\left(\hat{x}_{0}-x_{0}\right)+\alpha_{x y}^{(\hat{0})} \beta\left(\hat{x}_{0}\right) y_{0}\right] \frac{\alpha_{y x}^{(1)}}{\delta_{y}^{(2)}} \frac{1}{\alpha_{x x}^{(1)}+\alpha_{y x}^{(1)}+\delta_{x}^{(1)}} .
\end{aligned}
$$


This shows that, in the absence of the compensating term in $y_{0}$, a difference in $x_{0}$ will be reflected in a difference in both $x_{2}^{*}$ and $y_{2}^{*}$, and thus imply the occurrence respectively of CHD and diabetes. Thus the occurrence of each disease will be related to both the onset of the altered signal and to the change from the canonical pathway of its propagation downstream. Expressing $\hat{x}_{0}$ and $\hat{y}_{2}^{*}$ as functions of $\hat{x}_{2}^{*}$, we find that

$$
\begin{aligned}
& \hat{x}_{0}=\frac{1}{A_{0}}\left(A_{2} \hat{x}_{2}^{*}-A\right) \\
& \hat{y}_{2}^{*}=\frac{\hat{x}_{2}^{*}}{B_{2}}
\end{aligned}
$$

where the $A$ 's and $B$ 's are functions of the biochemical rates, the canonical signal $x_{0}$ and the fixed point of the canonical pathway $x_{2}^{*}$.

For case 1b)

$$
\begin{aligned}
\frac{d \hat{x}_{1}}{d t} & =\alpha_{x x}^{(0)} \hat{x}_{0}+\alpha_{x y}^{(0)} \beta\left(\hat{x}_{0}\right) y_{0}-\left(\alpha_{x x}^{(1)}+\delta_{x}^{(1)}\right) \hat{x}_{1} \\
\frac{d \hat{y}_{1}}{d t} & =\alpha_{y y}^{(0)}\left[1-\beta\left(\hat{x}_{0}\right)\right] y_{0}-\left(\alpha_{y y}^{(1)}+\delta_{y}^{(1)}\right) \hat{y}_{1} \\
\frac{d \hat{x}_{2}}{d t} & =\alpha_{x x}^{(1)} \hat{x}_{1}-\delta_{x}^{(2)} \hat{x}_{2} \\
\frac{d \hat{y}_{2}}{d t} & =\alpha_{y y}^{(1)} \hat{y}_{1}-\delta_{y}^{(2)} \hat{y}_{2} .
\end{aligned}
$$

The fixed points for the canonical and altered pathways are related as follows

$$
\begin{aligned}
& \hat{x}_{2}^{*}-x_{2}^{*}=\left[\alpha_{x x}^{(0)}\left(\hat{x}_{0}-x_{0}\right)+\alpha_{x y}^{(\hat{0})} \beta\left(\hat{x}_{0}\right) y_{0}\right] \frac{\alpha_{x x}^{(1)}}{\delta_{x}^{(2)}} \frac{1}{\alpha_{x x}^{(1)}+\delta_{x}^{(1)}} \\
& \hat{y}_{2}^{*}-y_{2}^{*}=-\alpha_{y y}^{(\hat{0})} \beta\left(\hat{x}_{0}\right) y_{0} \frac{\alpha_{y x}^{(1)}}{\delta_{y}^{(2)}} \frac{1}{\alpha_{y y}^{(1)}+\delta_{y}^{(1)}} .
\end{aligned}
$$

In this case, the change from the sharing of a component while compensating for the supply of one pathway could cause the other to be depleted of essential reagents. The outcome, however, will depend on how much the intercepting pathway takes and how much the intercepted pathway can run canonically without. Similarly we find expressions for $\hat{x}_{0}$ and $\hat{y}_{2}^{*}$ as functions of $\hat{x}_{2}^{*}$

$$
\begin{aligned}
& \hat{x}_{0}=\frac{1}{A_{0}}\left(A_{2} \hat{x}_{2}^{*}-A\right) \\
& \hat{y}_{2}^{*}=\frac{1}{B_{2}}\left[\left(A_{2} \hat{x}_{2}^{*}-A\right) \frac{B_{0}}{A_{0}}+B\right]
\end{aligned}
$$

where similarly the $A$ 's and $B$ 's are functions of the biochemical rates, the canonical signal $x_{0}$ and the fixed points of the canonical pathways $x_{2}^{*}$ and $y_{2}^{*}$.

In the following subsection we will use as the working description of the signalling pathway the dynamical system of case 1a). Given the similarity in the functional form of the dependence of the variables, similar conclusions would also be inferred, with the interpretation only differing on the basis of the different structure of the pathways. 


\section{B. Translation of the dynamical system into a probability description}

In order to compute $P(C H D \mid S N P \wedge H)$, we proceed to write the probabilities in Eqn. (13) in terms of the variables in the description of the dynamical system. In particular, we want to compute

$$
P\left(x_{2} \mid x_{0}\right)=\frac{P\left(x_{0} \mid x_{2}\right) P\left(x_{2}\right)}{P\left(x_{0}\right)}
$$

where the probability $P\left(x_{2}\right)$ is related to that for the occurrence of CHD and the likelihood $P\left(x_{0} \mid x_{2}\right)$ is related to that for the data on the SNP conditional on the prior for CHD. The quantity $P\left(x_{0}\right)$ is the evidence, which is found by marginalising the likelihood and is related to the probability for occurrence of the SNP. We will use the probabilities computed in the previous section to constrain the priors assumed here. We will then derive an expression for $P(C H D \mid S N P \wedge H)$ in terms of both the statistical properties of the priors and the biochemical parameters of the transmission process from the SNP to the CHD.

We assume that the probability for the occurrence of CHD is described by a Gaussian distribution with expectation value equal to the fixed point of the final component of the pathway $\mu_{2_{P}}=x_{2}^{*}$, and standard deviation $\sigma_{2_{P}}$

$$
P\left(x_{2}\right)=\frac{1}{\sqrt{2 \pi} \sigma_{2_{P}}} \exp \left[-\frac{\left(x_{2}-\mu_{2_{P}}\right)^{2}}{2 \sigma_{2_{P}}^{2}}\right] .
$$

In the probability description, the quantities $\mu_{2_{P}}$ and $\sigma_{2_{P}}$ are properties of the prior knowledge of the distribution of the occurrence of CHD derived from population sampling and expressed in terms of the biochemical parameters of the system according to the model considered. In the absence of further data, these quantities characterize a theoretical prior which we can assume to be approximated by a binomial distribution for sufficiently large $N_{C H D}$. The parameter of the binomial distribution is the frequency of occurrence of CHD

which has for maximal likelihood estimator $p_{C H D}=N_{C H D} /\left(N_{C H D}+N_{\overline{C H D}}\right)$. The mean and the variance of the approximated Gaussian distribution are given by

$$
\mu_{2_{P}}=N_{C H D} p_{C H D}, \quad \sigma_{2_{P}}^{2}=N_{C H D} p_{C H D}\left(1-p_{C H D}\right)
$$

which yield respectively $\mu_{2_{P}}=170 \pm 1$ and $\sigma_{2_{P}}=9.2 \pm 1.6$. The fixed point corresponding to the canonical pathway denotes absence of disease, whereas deviations from this value will entail a non-vanishing probability of occurrence of CHD. In order to quantify this probability, we need to devise a criterion to determine the emergence of CHD. Deviations on the fixed point of an altered pathway from that of the canonical pathway are quantified 
by $\Delta_{2}=\mu_{2_{P}}-\hat{x}_{2}^{*}{ }^{2}$ This quantity would measure a non-environmental marker ${ }^{3}$ for the occurrence of CHD [28]. For deviations larger than a threshold value $\Delta_{2}^{\text {thres }}$ the disease will occur. The probability of occurrence of CHD will be

$$
\begin{aligned}
P(C H D) & =P\left(x_{2}<\mu_{2_{P}}-\Delta_{2}^{\text {thres }}\right) \\
& =\frac{1}{\sqrt{2 \pi} \sigma_{2_{P}}} \int_{-\infty}^{\mu_{2_{P}}-\Delta_{2}^{\text {thres }}} d x_{2} \exp \left[-\frac{\left(x_{2}-\mu_{2_{P}}\right)^{2}}{2 \sigma_{2_{P}}^{2}}\right] .
\end{aligned}
$$

Deviations at the level of $x_{2}$ will be the result of the propagation along the pathway of deviations at the level of $x_{0}$ according to Eqn. (18) or (26), depending on the model considered.

The likelihood of the data on $x_{0}$ given the variable $x_{2}$ is assumed also to follow a Gaussian distribution centred at $\hat{x}_{0}$ and with standard deviation $\sigma_{0_{D}}$ [29]

$$
P\left(\hat{x}_{0} \mid \hat{x}_{2}^{*}\right)=\frac{1}{\sqrt{2 \pi} \sigma_{0_{D}}} \exp \left[-\frac{\left(\hat{x}_{0}-\hat{x}_{2}^{*}\right)^{2}}{2 \sigma_{0_{D}}^{2}}\right] .
$$

Here the quantities $\hat{x}_{0}$ and $\sigma_{0_{D}}$ describe properties of the data in the presence of the SNP expressed in terms of the biochemical parameters of the system. The likelihood of CHD will be given by the integral in $x_{2}$ because the SNP enters as data through the modelling of the system. Substituting $\hat{x}_{0}=\hat{x}_{0}\left(\hat{x}_{2}^{*}\right)=\left(A_{2} \hat{x}_{2}^{*}-A\right) / A_{0}$, where the $A$ 's are functions of the statistical parameters $\mu_{2_{P}}, x_{0}$ and $\sigma_{0_{D}}$ as well as of the biochemical parameters, we find that

$$
\begin{aligned}
P(S N P \mid C H D) & =\frac{1}{\sqrt{2 \pi} \sigma_{0_{D}}} \int_{-\infty}^{\mu_{2_{P}}-\Delta_{2}^{\text {thres }}} d \hat{x}_{2}^{*} \exp \left[-\frac{\left(\hat{x}_{0}-\hat{x}_{2}^{*}\right)^{2}}{2 \sigma_{0_{D}}^{2}}\right] \\
& =\frac{1}{2}+\frac{A_{0}}{2\left(A_{0}-A_{2}\right)} \operatorname{erf}\left[\frac{A+\left(\mu_{2_{P}}-\Delta_{2}^{\text {thres }}\right)\left(A_{0}-A_{2}\right)}{\sqrt{2} \sigma_{0_{D}} A_{0}}\right]
\end{aligned}
$$

where $\operatorname{erf}$ stands for the error function given by the integral erf $(x)=(2 / \sqrt{\pi}) \int_{0}^{x} d y \exp \left[-y^{2}\right]$. This probability was computed in Eqn. (11).

We can now derive the functional form of the evidence in terms of the statistical and the biochemical parameters. Combining the two assumptions above, we find that

$$
P\left(\hat{x}_{0} \mid \hat{x}_{2}^{*}\right) P\left(\hat{x}_{2}^{*}\right)=\frac{1}{2 \pi \sigma_{0_{D}} \sigma_{2_{P}}} \exp \left[-\frac{1}{2}\left(\Gamma-\mu_{e f f}^{2}\right)\right] \exp \left[-\frac{\left(\hat{x}_{2}^{*}-\mu_{e f f}\right)^{2}}{2 \sigma_{e f f}^{2}}\right]
$$

2 In accordance with the description encapsulated in the model, the SNP will act by causing deficiency in the modus operandi of the system. Should it instead act by causing excess, then a symmetric interval about $\mu_{2_{P}}$ would be the generalization to account for possible saturation and consequent screening effect. The changes to implement in all the subsequent results would be straightforward.

3 The distinction between functional and environmental markers can be shady and will thus require care. 
where

$$
\begin{aligned}
& \mu_{e f f}=\left(\frac{\mu_{2_{P}}}{\sigma_{2_{P}}^{2}}-\frac{\hat{x}_{0}}{\sigma_{0_{D}}^{2}}\right) /\left(\frac{1}{\sigma_{2_{P}}^{2}}+\frac{1}{\sigma_{0_{D}}^{2}}\right), \\
& \sigma_{\text {eff }}^{2}=1 /\left(\frac{1}{\sigma_{2_{P}}^{2}}+\frac{1}{\sigma_{0_{D}}^{2}}\right), \\
& \Gamma=\left(\frac{\mu_{2_{P}}^{2}}{\sigma_{2_{P}}^{2}}+\frac{\hat{x}_{0}^{2}}{\sigma_{0_{D}}^{2}}\right) .
\end{aligned}
$$

Substituting $\hat{x}_{0}=\hat{x}_{0}\left(\hat{x}_{2}^{*}\right)$, we integrate in $\hat{x}_{2}^{*}$ finding that

$$
\begin{aligned}
& P(S N P)=\int_{-\infty}^{+\infty} d \hat{x}_{2}^{*} P\left(\hat{x}_{0} \mid \hat{x}_{2}^{*}\right) P\left(\hat{x}_{2}^{*}\right) \\
= & \frac{1}{\sqrt{2 \pi}} \frac{1}{\sqrt{\sigma_{0_{D}}^{2}+\sigma_{2_{P}}^{2}\left(A_{0}-A_{2}\right)^{2} / A_{0}^{2}}} \exp \left[-\frac{1}{2} \frac{\left[A / A_{0}+\mu_{2_{P}}\left(A_{0}-A_{2}\right) / A_{0}\right]^{2}}{\sigma_{0_{D}}^{2}+\sigma_{2_{P}}^{2}\left(A_{0}-A_{2}\right)^{2} / A_{0}^{2}}\right] .
\end{aligned}
$$

If we furthermore assume that $x_{0}$ follows a Gaussian distribution centred at the value for the canonical path, which we denote by $\mu_{0}$, and with standard deviation $\sigma_{0_{D}}$, which is such that $\Delta_{0}^{\text {thres }}=\sqrt{2 \sigma_{0_{D}}^{2} \ln \left[1 / P\left(\hat{x}_{0}\right)\right]}$ when $\hat{x}_{0}=\mu_{0}-\Delta_{0}^{\text {thres }}$, then following a similar reasoning to that for CHD, we will have presence of the SNP for $\hat{x}_{0}<\mu_{0}-\Delta_{0}^{\text {thres }}$. Hence

$$
P(S N P)=\frac{1}{\sqrt{2 \pi} \sigma_{0_{D}}} \int_{-\infty}^{\mu_{0}-\Delta_{0}^{\text {thres }}} d \hat{x}_{0} \exp \left[-\frac{\left(\hat{x}_{0}-\mu_{0}\right)^{2}}{2 \sigma_{0_{D}}^{2}}\right]=\frac{1}{2}-\frac{1}{2} \operatorname{erf}\left[\frac{\Delta_{0}^{\text {thres }}}{\sqrt{2} \sigma_{0_{D}}}\right]
$$

which equals $\lambda_{\text {eff }}=0.30 \pm 0.001$, as computed in Eqn. (9), and thus serves to constrain the parameters in Eqn. (40). We can also solve for $\Delta_{0}^{\text {thres }}$ finding that $\Delta_{0}^{\text {thres }}=(0.52 \pm 0.006) \sigma_{0_{D}}$. The quantity $\Delta_{0}^{\text {thres }}$ determines the parameter $\beta$ in Eqn. (17). Combining the two conditions above, we find the value for $P\left(\hat{x}_{0}\right)=0.87 \pm 0.003$ which we can interpret as the probability that the SNP has occurred when $\hat{x}_{0}$ is below the threshold value that can trigger the canonical pathway.

Moreover, having in Eqn. (12) also computed $P(t 2 D M \mid C H D)$, we write the corresponding likelihood

$$
P\left(\hat{y}_{2}^{*} \mid x_{2}^{*}\right)=\frac{1}{\sqrt{2 \pi} s_{2_{D}}} \exp \left[-\frac{\left(\hat{y}_{2}^{*}-\hat{x}_{2}^{*}\right)^{2}}{2 s_{2_{D}}^{2}}\right] .
$$

Note that we cannot follow a reasoning analogous to that for the case of the probability of $C H D$ because the population $N_{t 2 D M}$ is not entirely random. Substituting $\hat{y}_{2}^{*}=\hat{y}_{2}^{*}\left(\hat{x}_{2}^{*}\right)=$ $\hat{x}_{2}^{*} / B_{2}$, where $B_{2}$ is a function of the biochemical parameters, we find that

$$
\begin{aligned}
P(t 2 D M \mid C H D) & =\frac{1}{\sqrt{2 \pi} s_{2_{D}}} \int_{-\infty}^{\mu_{2_{P}}-\Delta_{2}^{\text {thres }}} d \hat{x}_{2}^{*} \exp \left[-\frac{\left(\hat{y}_{2}^{*}-\hat{x}_{2}^{*}\right)^{2}}{2 s_{2_{D}}^{2}}\right] \\
& =\frac{1}{2}+\frac{B_{2}}{2\left(1-B_{2}\right)} \operatorname{erf}\left[\frac{\left(\mu_{2_{P}}-\Delta_{2}^{t h r e s}\right)\left(1-B_{2}\right)}{\sqrt{2} s_{2_{D}} B_{2}}\right] .
\end{aligned}
$$


We can now compute the posterior probability of the variable $x_{2}$ given $\hat{x}_{0}$, i.e.

$$
P\left(\hat{x}_{2}^{*} \mid \hat{x}_{0}\right)=\frac{1}{2 \pi \sigma_{0_{D}} \sigma_{2_{P}}} \frac{1}{P\left(\hat{x}_{0}\right)} \exp \left[-\frac{1}{2}\left(\Gamma-\mu_{e f f}^{2}\right)\right] \exp \left[-\frac{\left(\hat{x}_{2}^{*}-\mu_{e f f}\right)^{2}}{2 \sigma_{e f f}^{2}}\right],
$$

finding for the probability that CHD will occur given that SPN has occurred that

$$
\begin{aligned}
& P(C H D \mid S N P)=\frac{1}{P(S N P)} \int_{-\infty}^{\mu_{2}{ }^{-\Delta_{2}^{\text {thres }}}} d \hat{x}_{2}^{*} P\left(\hat{x}_{0} \mid \hat{x}_{2}^{*}\right) P\left(\hat{x}_{2}^{*}\right) \\
= & \frac{1}{2}+\frac{1}{2} \operatorname{erf}\left[\frac{-\sigma_{0_{D}}^{2} \Delta_{2}^{\text {thres }}+\sigma_{2_{P}}^{2}\left[A+\left(\mu_{2_{P}}-\Delta_{2}\right)\left(A_{0}-A_{2}\right)\right]\left(A_{0}-A_{2}\right) / A_{0}^{2}}{\sqrt{2} \sigma_{0_{D}} \sigma_{2_{P}} \sqrt{\sigma_{0_{P}}^{2}+\sigma_{2_{P}}^{2}\left(A_{0}-A_{2}\right)^{2} / A_{0}^{2}}}\right] .
\end{aligned}
$$

The variables in this formal expression are constrained by the relations found above and which we summarize below:

$$
\begin{aligned}
P(S N P) & \equiv f_{00}\left(x_{0}, \sigma_{0_{D}} ; \alpha_{i j}^{(k)}, \beta\right)=0.30 \pm 0.001 \\
P(S N P \mid C H D) & \equiv f_{02}\left(x_{0}, \sigma_{0_{D}}, \Delta_{2}^{\text {thres }} ; \alpha_{i j}^{(k)}, \beta\right)=0.20 \pm 0.001 \\
P(t 2 D M \mid C H D) & \equiv f_{22}\left(s_{2_{D}}, \Delta_{2}^{\text {thres }} ; \alpha_{i j}^{(k)}, \beta\right)=0.09 \pm 0.001 .
\end{aligned}
$$

Here the subscript $D$ indicates properties of the data as derived form the model and constrained by these particular data, and the subscript $P$ indicates properties of the prior which are based on the knowledge inferred from data sets delivered by other experiments. The results in Eqns. (46) and (47) are of the same order as relative proportions found in other studies, respectively in Ref. [4] and Refs. [3, 11]. The result in Eqn. (12) is a result of this study.

These functions depend on our knowledge of the rates in the model of the implicated pathways as well as on the statistical properties of the associated risk factor. However, from these three relations as constrained by the data, we can solve for three parameters

only. Solving for the remaining parameters requires additional conditions for the statistical properties of the priors and biochemical parameters. Nonetheless, the idea that the present study serves to introduce and which we here applied to one data set on one risk factor has been demonstrated, i.e. a) how to extract the statistical properties of the event from the corresponding phenomenological data and then b) from the statistical properties of the event how to extract biochemical information on the causal relations that link the event with the risk factor.

\section{DISCUSSION}

In this manuscript we derive the probability of occurrence of CHD based on data in the presence of the SNP at the -308 position of the TNF- $\alpha$ gene. We first worked following a 
bottom-up approach. Comparing different hypotheses for the statistical relation between the occurrences of SNP and CHD, we selected the working hypothesis on the basis of the Bayes factors. We showed that the data favour (although without strong evidence) the association of the SNP with the occurrence of CHD as well as the participation of the occurrence of t2DM in the causal relation. Using the Bayes theorem, we computed the probability of the SNP conditional on the occurrence of CHD. We then worked following a top-down approach. We presented a schematic model for a simplistic description of the signalling pathway which relates the presence of the SNP with the emergence of CHD. The data contain information on equilibrium states of the several variables that describe the biochemical system and can thus be translated into a probability description. We then computed the probability of CHD given that the SNP had occurred, using for the likelihood the probability previously computed. We expressed the result as a function of both the biochemical parameters of the model and the statistical parameters of the prior probability distributions. Other probabilities were also computed, which serve as constraints to the parameters.

In an upcoming study we will be exploring the idea further by integrating the sparse existing data on various population samplings. We will select the data for CHD given different risk factors, and for the SNP given different diseases. From the first selection we intend to extract the remaining statistical parameters, since the prior of CHD will be shared. Also a link should be established between this formalism and the CHD prediction estimates from a multivariable risk calculation [30]. From the second selection we intend to extract the biochemical parameters of the signalling pathway. Although the prior of CHD will be shared, the biochemical system will grow in complexity and new rates will be introduced. We expect, however, that by exhausting the data sets available we will reach a balance of unknowns and equations that would allow us to solve the problem. Should this balance not be attained, we will resort to determining confidence levels for the unknown parameters based on simulations [18 20]. Whenever available, we will complement the study with temporal information to obtain reaction rates [31]. Ultimately we expect to be able to infer a universal law for gene mutation by systematising the various diseases into a comprehensive model of the signalling pathway.

\section{Acknowledgments}

CSC is supported by Fundação para a Ciência e a Tecnologia (FCT), SFRH/BPD/65993/2009. The authors thank AM Teixeira for a careful reading of the manuscript and PC Aguiar, ME Chollet and Z Geitona for insightful comments. CSC

also acknowledges the hospitality of the Astrophysical Sciences Department, Princeton University. 


\section{Appendix A: Computation of the evidence}

In this Appendix we compute the evidence for the six hypotheses discussed in Section II] Hypothesis $H_{00}$ has only one free parameter, the probability that the SNP occurred. This probability, describing a mutation process, is assumed to have a Poisson distribution characterized by the size of the population $N$ and a mutation rate $\lambda$. The probability of $n$ mutations is

$$
P(n \mid \lambda \wedge N)=\exp [-\lambda N] \frac{(\lambda N)^{n}}{n !}
$$

with the mean number $\langle n\rangle=\lambda N$. For $n=N_{S N P}$ mutations in a sample of size $N$ and a uniform prior distribution for $\lambda, P(\lambda)=1$, we find that

$$
\begin{aligned}
P\left(D_{S N P} \mid H_{00}\right) & =\int_{0}^{\infty} d \lambda P\left(D_{S N P} \mid \lambda \wedge N\right) P\left(\lambda \mid H_{00}\right) \\
& =\int_{0}^{\infty} d \lambda \exp [-\lambda N] \frac{(\lambda N)^{N_{S N P}}}{N_{S N P} !}=\frac{1}{N} .
\end{aligned}
$$

Hypothesis $H_{01}$ has two parameters, the probabilities that the SNP occurred given the two values of the variable $C H D$. There are two possible sources of SNP, namely the population with CHD and the population without CHD. The presence of the SNP follows a binomial distribution where $p_{01}$ is the frequency of the SNP for the case of $C H D$ and $p_{0 \overline{1}}$ is the frequency of the SNP for the case of $\overline{C H D}$. It follows that

$$
\begin{aligned}
P\left(D_{S N P} \mid H_{01}\right)= & \int d p_{01} \int d p_{0 \overline{1}} P\left(D_{S N P} \mid p_{01} \wedge p_{0 \overline{1}} \wedge H_{01}\right) P\left(p_{01} \wedge p_{0 \overline{1}} \mid H_{01}\right) \\
= & \int d p_{01} P\left(D_{S N P} \mid p_{01} \wedge H_{01}\right) P\left(p_{01} \mid H_{01}\right) \\
& +\int d p_{0 \overline{1}} P\left(D_{S N P} \mid p_{0 \overline{1}} \wedge H_{01}\right) P\left(p_{0 \overline{1}} \mid H_{01}\right) .
\end{aligned}
$$

Moreover, assuming a uniform prior distribution probability for the frequencies $p_{01}$ and $p_{0 \overline{1}}$ of the data on the SNP given respectively the occurrence or non-occurrence of CHD

$$
P\left(p_{01} \mid H_{01}\right)=1, \quad P\left(p_{0 \overline{1}} \mid H_{01}\right)=1,
$$

we find that

$$
\begin{aligned}
P\left(D_{S N P} \mid H_{01}\right)= & \int d p_{01}\left(\begin{array}{c}
N_{C H D} \\
N_{S N P, C H D}
\end{array}\right) p_{01}^{N_{S N P, C H D}}\left(1-p_{01}\right)^{N_{\overline{S N P}, C H D}} \\
& +\int d p_{0 \overline{1}}\left(\begin{array}{c}
N_{\overline{C H D}} \\
N_{S N P, \overline{C H D}}
\end{array}\right) p_{0 \overline{1}}^{N_{S N P, \overline{C H D}}}\left(1-p_{0 \overline{1}}\right)^{N_{\overline{S N P}, \overline{C H D}}} \\
= & \left(\begin{array}{c}
N_{C H D} \\
N_{S N P, C H D}
\end{array}\right) \frac{N_{S N P, C H D} ! N_{\overline{S N P}, C H D} !}{\left(N_{C H D}+1\right) !} \\
& +\left(\begin{array}{c}
N_{\overline{C H D}} \\
N_{S N P, \overline{C H D}}
\end{array}\right) \frac{N_{S N P, \overline{C H D}} ! N_{\overline{S N P}, \overline{C H D}} !}{\left(N_{\overline{C H D}}+1\right) !}
\end{aligned}
$$




$$
=\frac{1}{N_{C H D}+1}+\frac{1}{N_{\overline{C H D}}+1} .
$$

Similarly to $H_{01}$, hypothesis $H_{10}$ has two parameters, the probabilities that the SNP occurred given the two values of the variable $t 2 D M$. The evidence is given by the same expression as that of hypothesis $H_{01}$ with the variable $C H D$ replaced by the variable $t 2 D M$ and under the analogous assumptions on the corresponding frequency priors $p_{10}$ and $p_{\overline{1} 0}$.

Hypothesis $H_{11}$ has four parameters, one for each state of the variables $C H D$ and $t 2 D M$. This hypothesis combines the two hypotheses previously discussed which are assumed complementary, thus being a case of a two-component hypothesis with probabilities $\beta$ and $(1-\beta)$ [24. It follows that

$$
\begin{aligned}
P\left(D_{S N P} \mid H_{11}\right)= & \int d p_{01} \int d p_{10}\left[\beta P\left(D_{S N P} \mid p_{01} \wedge H_{11}\right) P\left(p_{01} \mid H_{11}\right)\right. \\
& \left.+(1-\beta) P\left(D_{S N P} \mid p_{10} \wedge H_{11}\right) P\left(p_{10} \mid H_{11}\right)\right] \\
+ & \int d p_{0 \overline{1}} \int d p_{\overline{1} 0}\left[\tilde{\beta} P\left(D_{S N P} \mid p_{0 \overline{1}} \wedge H_{11}\right) P\left(p_{0 \overline{1}} \mid H_{11}\right)\right. \\
& \left.+(1-\tilde{\beta}) P\left(D_{S N P} \mid p_{\overline{1} 0} \wedge H_{11}\right) P\left(p_{\overline{1} 0} \mid H_{11}\right)\right]
\end{aligned}
$$

which yields

$$
\begin{aligned}
P\left(D_{S N P} \mid H_{11}\right)= & \beta\left(\begin{array}{c}
N_{C H D} \\
N_{S N P, C H D}
\end{array}\right) \frac{N_{S N P, C H D} ! N_{\overline{S N P}, C H D} !}{\left(N_{C H D}+1\right) !} \\
& +(1-\beta)\left(\begin{array}{c}
N_{t 2 D M} \\
N_{S N P, t 2 D M}
\end{array}\right) \frac{N_{S N P, t 2 D M} ! N_{\overline{S N P}, t 2 D M} !}{\left(N_{t 2 D M}+1\right) !} \\
& +\tilde{\beta}\left(\begin{array}{c}
N_{\overline{C H D}} \\
N_{S N P, \overline{C H D}}
\end{array}\right) \frac{N_{S N P, \overline{C H D}} ! N_{\overline{S N P}, \overline{C H D}} !}{\left(N_{\overline{C H D}}+1\right) !} \\
& +(1-\tilde{\beta})\left(\begin{array}{c}
N_{\overline{t 2 D M}} \\
N_{S N P, \overline{t 2 D M}}
\end{array}\right) \frac{N_{S N P, \overline{t 2 D M}} ! N_{\overline{S N P}, \overline{t 2 D M}} !}{\left(N_{\overline{t 2 D M}}+1\right) !} \\
= & \beta \frac{1}{N_{C H D}+1}+(1-\beta) \frac{1}{N_{t 2 D M}+1} \\
& +\tilde{\beta} \frac{1}{N_{\overline{C H D}}+1}+(1-\tilde{\beta}) \frac{1}{N_{\overline{t 2 D M}}+1} .
\end{aligned}
$$

Here $\beta=N_{C H D} /\left(N_{C H D}+N_{t 2 D M}\right)$ is the probability that the data were extracted from the pool of hypothesis $H_{01}$ and $\tilde{\beta}=N_{\overline{C H D}} /\left(2 N_{\overline{C H D}, \overline{t 2 D M}}+N_{C H D, \overline{t 2 D M}}+N_{\overline{C H D}, t 2 D M}\right)$ the probability that the pool is that of the complement of $H_{01}$. Similarly we define $(1-\beta)$ and $(1-\tilde{\beta})$ from hypothesis $H_{10}$.

For Hypothesis $H_{11}^{c h d}$ we have four parameters for combined states of the variables $C H D$ and $t 2 D M$, namely $C H D \wedge \overline{t 2 D M}$ and $\overline{C H D} \wedge \overline{t 2 D M}$ as the states which are conditional to non-occurrence of $\mathrm{t} 2 \mathrm{DM}$, and $t 2 D M \wedge C H D$ and $\overline{t 2 D M} \wedge C H D$ as the states which are conditioned to occurrence of CHD. The corresponding four frequencies are as follows: $\tilde{p}_{01}$ is the frequency of SNP given the occurrence of CHD and $\tilde{p}_{0 \overline{1}}$ the frequency of SNP given non-occurrence of CHD, both subject to non-occurrence of $\mathrm{t} 2 \mathrm{DM} ; \tilde{p}_{11}$ is the frequency of 
SNP given that t2DM has occurred and $\tilde{p}_{\overline{1} 1}$ the frequency of SNP given that t2DM has not occurred, both subject to $C H D$ having occurred. For a uniform prior probability of these frequencies, we find that

$$
\begin{aligned}
P\left(D_{S N P} \mid H_{11}^{\text {chd }}\right)= & \int d \tilde{p}_{01} \int d \tilde{p}_{11}\left[\gamma P\left(D_{S N P} \mid \tilde{p}_{01} \wedge H_{11}^{\text {chd }}\right) P\left(\tilde{p}_{01} \mid H_{11}^{\text {chd }}\right)\right. \\
& \left.+(1-\gamma) P\left(D_{S N P} \mid \tilde{p}_{11} \wedge H_{11}^{\text {chd }}\right) P\left(\tilde{p}_{11} \mid H_{11}^{\text {chd }}\right)\right] \\
+ & \int d \tilde{p}_{0 \overline{1}} \int d \tilde{p}_{\overline{1} 1}\left[\tilde{\gamma} P\left(D_{S N P} \mid \tilde{p}_{0 \overline{1}} \wedge H_{11}^{\text {chd }}\right) P\left(\tilde{p}_{0 \overline{1}} \mid H_{11}^{\text {chd }}\right)\right. \\
& \left.+(1-\tilde{\gamma}) P\left(D_{S N P} \mid \tilde{p}_{\overline{1} 1} \wedge H_{11}^{\text {chd }}\right) P\left(\tilde{p}_{\overline{1} 1} \mid H_{11}^{\text {chd }}\right)\right]
\end{aligned}
$$

which yields

$$
\begin{aligned}
& P\left(D_{S N P} \mid H_{11}^{\text {chd }}\right)=\gamma\left(\begin{array}{c}
N_{C H D, \overline{t 2 D M}} \\
N_{S N P, C H D, \overline{t 2 D M}}
\end{array}\right) \frac{N_{S N P, C H D \overline{t 2 D M}} ! N_{\overline{S N P}, C H D, \overline{t 2 D M}} !}{\left(N_{C H D, \overline{t 2 D M}}+1\right) !} \\
& +(1-\gamma)\left(\begin{array}{c}
N_{C H D, t 2 D M} \\
N_{S N P, C H D, t 2 D M}
\end{array}\right) \frac{N_{S N P, C H D, t 2 D M} ! N_{\overline{S N P}, C H D, t 2 D M} !}{\left(N_{C H D, t 2 D M}+1\right) !} \\
& +\tilde{\gamma}\left(\begin{array}{c}
N_{\overline{C H D}, \overline{t 2 D M}} \\
N_{S N P, \overline{C H D}, \overline{t 2 D M}}
\end{array}\right) \frac{N_{S N P, \overline{C H D}, \overline{t 2 D M}} ! N_{\overline{S N P}, \overline{C H D}, \overline{t 2 D M}} !}{\left(N_{\overline{C H D}, \overline{t 2 D M}}+1\right) !} \\
& +(1-\tilde{\gamma})\left(\begin{array}{c}
N_{C H D, \overline{t 2 D M}} \\
N_{S N P, C H D, \overline{t 2 D M}}
\end{array}\right) \frac{N_{S N P, C H D, \overline{t 2 D M}} ! N_{\overline{S N P}, C H D, \overline{t 2 D M}} !}{\left(N_{C H D, \overline{t 2 D M}}+1\right) !} \\
& =\gamma \frac{1}{N_{C H D, \overline{t 2 D M}}+1}+(1-\gamma) \frac{1}{N_{C H D, t 2 D M}+1} \\
& +\tilde{\gamma} \frac{1}{N_{\overline{C H D}, \overline{t 2 D M}}+1}+(1-\tilde{\gamma}) \frac{1}{N_{C H D, \overline{t 2 D M}}+1} .
\end{aligned}
$$

Here $\gamma=N_{C H D, \overline{t 2 D M}} / N_{C H D}$ and $\tilde{\gamma}=N_{\overline{C H D} \overline{t 2 D M}} / N_{\overline{t 2 D M}}$.

Similarly to $H_{11}^{(c h d)}$, hypothesis $H_{11}^{(t 2 d m)}$ has four parameters for combined states of the variables $C H D$ and $t 2 D M$, namely $t 2 D M \wedge \overline{C H D}$ and $\overline{t 2 D M} \wedge \overline{C H D}$ as the states conditional on non-occurrence of CHD, and $C H D \wedge t 2 D M$ and $\overline{C H D} \wedge t 2 D M$ as the states conditional on occurrence of t2DM. The frequencies are analogously defined to those of hypothesis $H_{11}^{(c h d)}$. The evidence is given by the same expression as that of hypothesis $H_{11}^{(\text {chd })}$ with $\tilde{p}_{01}$ and $\tilde{p}_{0 \overline{1}}$ replaced by the frequency of SNP, subject to non-occurrence of CHD, given the occurrence or non-occurrence of t2DM respectively $\tilde{p}_{10}$ and $\tilde{p}_{\overline{1} 0}$, and $\tilde{p}_{\overline{1} 1}$ replaced by $\tilde{p}_{1 \overline{1}}$. Analogously $\gamma$ is replaced by $N_{\overline{C H D}, t 2 D M} / N_{t 2 D M}$ and $\tilde{\gamma}$ by $N_{\overline{C H D}, \overline{t 2 D M}} / N_{\overline{C H D}}$.

\section{Appendix B: Calculation of $P\left(S N P \mid H_{00}\right)$}

In this Appendix we compute for the purpose of comparison the probability of occurrence of SNP for hypothesis $H_{00}$. Starting from Eqn. (A1) and using the Bayes theorem, we find for the posterior probability of $\lambda$ that

$$
P\left(\lambda \mid D_{S N P} \wedge N\right)=\frac{P\left(D_{S N P} \mid \lambda \wedge N\right) P(\lambda \mid N)}{P\left(D_{S N P} \mid N\right)} .
$$


The normalizing constant is the evidence computed in Eqn. A2). Given the data and for $P\left(S N P \mid \lambda \wedge H_{00}\right)=\lambda$, the probability of a mutation in a population of size $N$ is

$$
\begin{aligned}
P\left(S N P \mid H_{00}\right) & =\int d \lambda P\left(S N P \mid \lambda \wedge H_{00}\right) P\left(\lambda \mid D_{S N P} \wedge H_{00}\right) \\
& =\int d \lambda \lambda N \exp [-\lambda N] \frac{(\lambda N)^{N_{S N P}}}{N_{S N P} !}=\frac{N_{S N P}+1}{N}
\end{aligned}
$$

which for $N_{S N P}=184$ and $N=683$ yields $P\left(S N P \wedge H_{00}\right)=0.27$. Despite the small difference between the values derived from the two hypotheses (which might be considered insignificant given the observational errors which are of order $1 / \sqrt{N} \approx 0.038$ ), the fact that there is a difference highlights the relevance of hypothesis testing before committing to a probability which will act as likelihood in subsequent calculations.

[1] Vassali P, The pathophysiology of tumor necrosis factors, Annu. Rev. Immunol 10 (1992) 411

[2] Vendrell J, Fernandez-Real J-M, Gutierrez C., Zamora A, Simon I, Bardaji A, Ricart W and Richart C, A polymorphism in the promoter of the tumor necrosis factor- $\alpha$ gene (-308) is associated with coronary disease in type 2 diabetic patients, Atherosclerosis 167 (2003) 257

[3] Dedoussis GV, Panagiotakos DB, Vidra NV, Louizou E, Chrysohoou C, Germanos A, Mantas Y, Tokmakidis S, Pitsavos C and Stefanadis C, Association between TNF-alpha -308G >A polymorphism and the development of acute coronary syndromes in Greek subjects: the CARDIO2000-GENE Study, Genet Med. 7(6) (2005) 411

[4] Elahi MM, Gilmour A, Matata BM and Mastana SS, A variant of position -308 of the Tumor necrosis factor alpha gene promoter and the risk of coronary heart disease, Heart Lung Circ. 17(1) (2008) 14

[5] Vourvouhaki E and Dedoussis GV, Cholesterol ester transfer protein: a therapeutic target in atherosclerosis? Expert Opin. Ther. Targets 12 (2008) 937

[6] Westendorp RGJ, Langermans JAM, Huizinga TWJ, Elouali AH, Verweij CL, Boomsma DI and Vandenbrouckke JP, Genetic influence on cytokine production and fatal menigococcal disease, Lancet 349 (1997) 170

[7] Abraham LJ and Kroeger KM, Impact of the -308 TNF promoter polymorphism on the transcriptional regulation of the TNF gene: relevance to disease, J Leukoc Biol 66 (1999) 562

[8] Wilson AG, di Giovine FS, Blakemore AI and Duff GW, Single base polymorphism in the humor tumour necrosis factor alpha (TNF alpha) gene detectable by NcoI restriction of PCR product, Hum Mol Genet 1 (1992) 353]

[9] Walston J, Seibert M, Yen CJ, Cheskin LJ and Andersen RE, Tumor necrosis factor-alpha-238 and -308 polymorphisms do not associate with traits related to obesity and insulin resistance, Diabetes 48 (1999) 2096 
[10] Hoffstedt J, Eriksson P, Hellstrom L, Rossner S, Ryden M and Arner P, Excessive fat accumulation is associated with the TNF alpha-308 G/A promoter polymorphism in women but not in men, Diabetologia 43 (2000) 117

[11] Koch W, Kastrati A, Bottiger C, Mehilli J, von Beckerath N and Schomig A, Interleukin10 and tumor necrosis factor gene polymorphisms and risk of coronary artery disease and myocardial infarction, Atherosclorosis 159 (2001) 137

[12] Cancello R, Tounian A, Poitou CH and Clement K, Adiposity signals, genetic and body weight regulation in humans, Diabetes Metab. 30 (2004) 215

[13] Stephens M and Balding DJ, Bayesian statistical methods for genetic association studies, Nature Reviews 10 (2009) 681

[14] Brewer BJ and Lewis GF, Strong Gravitational Lens Inversion: A Bayesian Approach, Astrophys. J. 637 (2006) 608-619 (arXiv:astro-ph/0509863v1)

[15] Trotta T, Applications of Bayesian model selection to cosmological parameters, Mon. Not. Roy. Astron. Soc. 378 (2007) 72 (arXiv:astro-ph/0504022 v3)

[16] Bridges M, Feroz F, Hobson MP and Lasenby AN, Bayesian optimal reconstruction of the primordial power spectrum (arXiv:0812.3541 v1 [astro-ph])

[17] Kiam S, Imoto S and Miyano S, Dynamic Bayesian network and nonparametric regression for non-linear modeling of gene network from time series gene expression data, BioSystems 75 (2004) 57

[18] Vyshemirsky V and Girolami MA, Bayesian Ranking of Biochemical System Models, Bioinformatics 24(6) (2008) 833

[19] Toni T, Welch D, Strelkowa N, Ipsen A and Stumpf MPH, Approximate Bayesian computation scheme for parameter inference and model selection in dynamical systems, J. Royal Society Interface, 6, 31 (2009) 187 (arXiv:0901.1925v1 [stat.CO])

[20] Toni T and Stumpf PH, Parameter inference and model selection in signaling pathway models (arXiv:0904.4468v1 [q-bio.QM])

[21] MacKay DJC, Information Theory, Inference, and Learning Algorithms, Cambridge University Press, 2003

[22] Kass RE, Raftery AE, Bayes Factors, J. American Statistical Association 90, 430 (1995) 773

[23] Frank AF, The Common Patterns of Nature (arXiv:0906:3597v1 [q-bio.QM])

[24] Guglielmetti F, Fischer R and Dose V, Background-source separation in astronomical images with Bayesian probability theory (I): the method (arXiv:0903.2342 [astro-ph.IM])

[25] Komarova NL, Zou X, Nie Q and Bardwell L, A theoretical framework for specificity in cell signalling, Mol. Systems Biology 4100031 (2005)

[26] Bardwell L, X. Zou, Nie Q and Komarova NL, Mathematical Models of Specificity in Cell Signaling, Biophys. J. 92 (2007) 3425

[27] Wodarz D and Komarova NL, Computational Biology of Cancer, Lecture Notes and Mathematical Modeling, World Scientific, 2005 
[28] Robinson SD, Dawson P, Ludlam CA, Boon NA and Newby DE, Vascular and fibrinolytic effects of intra-arterial tumour necrosis factor- $\alpha$ in patients with coronary heart disease, Clinical Science 110 (2006) 353

[29] Lupton R, Statistics in Theory and Practice, Princeton University Press, 1993

[30] Wilson PWF, D'Agostino RB, Levy D, Belanger AM, Silbershatz H and Kannel WB, Prediction of Coronary Heart Disease Using Risk Factor Categories, Circulation 97 (1998) 1837

[31] Vourvouhaki E, Carvalho C and Aguiar P, Model for Osteosarcoma-9 as a Potent Factor in Cell Survival and Resistance to Apoptosis, Phys.Rev. E76 (2007) 011926 (arXiv:0608030 [q-bio.SC]) 